\title{
A CHARACTERIZATION OF THE GAMMA DISTRIBUTION
}

\author{
GÉRARD LETAC,* Université Paul Sabatier
}

The aim of this note is to prove the following characterization.

Theorem. The positive number $\alpha$ is fixed, $X$ and $Y$ are two positive independent random variables and the distribution of $Y$ is defined by

$$
\mathbb{E}\left(Y^{s}\right)=\left(1+\frac{s}{\alpha}\right)^{\alpha+s} \text { for } s>0
$$

Then $X \exp (-X / \alpha) Y$ and $X$ have the same distribution if and only if the distribution of $X$ is

$$
\gamma_{\alpha}(d x)=\exp (-x) x^{\alpha-1} \mathbb{1}_{(0, \infty)}(x) \frac{d x}{\Gamma(\alpha)} .
$$

Klamkin [3] and Donald J. Newman (quoted in [3]) have made the following observation: if $U$ and $V$ are independent random variables with uniform distribution in $[0,1]$, then $(U V)^{U V}$ and $U^{U}$ have the same distribution. Writing $X=-\log U$ and $Y=-\log V$, a translation of this result is: if $X$ and $Y$ are independent random variables with distribution $\gamma_{1}$, then $(X+Y) \exp (-(X+Y))$ and $X \exp (-X)$ have the same distribution. Observing that $X+Y=X_{1}$ has distribution $\gamma_{2}$, this result admits an easy extension, as follows.

Proposition 1. The positive number $\alpha$ is fixed, $X$ and $X_{1}$ have respective distributions $\gamma_{\alpha}$ and $\gamma_{\alpha+1}$. Then:

(1) $X \exp (-X / \alpha)$ and $X_{1} \exp \left(-X_{1} / \alpha\right)$ have the same distribution

(2) $\mathbb{E}\left((X \exp (-X / \alpha))^{s}\right)=\frac{\Gamma(\alpha+s)}{\Gamma(\alpha)}\left(1+\frac{s}{\alpha}\right)^{-(\alpha+s)}$ if $s>-\alpha$.

Proof. Taking $s>-\alpha$, one gets (2) by

$$
\mathbb{E}\left((X \exp (-X / \alpha))^{s}\right)=\int_{0}^{\infty} x^{s+\alpha-1} \exp \left(-x\left(1+\frac{s}{\alpha}\right)\right) \frac{d x}{\Gamma(\alpha)} .
$$

In the same way

$$
\mathbb{E}\left(\left(X_{1} \exp \left(-X_{1} / \alpha\right)\right)^{2}\right)=\frac{\Gamma(\alpha+1+s)}{\Gamma(\alpha+1)}\left(1+\frac{s}{\alpha}\right)^{-(\alpha+1+s)}
$$

which is $\mathbb{E}\left((X \exp (-X / \alpha))^{s}\right)$ by using $\Gamma(z+1)=z \Gamma(z)$; this proves (1).

Received 18 April 1985.

* Postal address: Laboratoire de Statistique et Probabilités, UA-CNRS 745, Université Paul Sabatier, 118 route de Narbonne, 31062 Toulouse Cedex, France. 
The interesting point in (2) is that $\mathbb{E}\left(X^{s}\right)=\Gamma(\alpha+s) / \Gamma(\alpha)$. Therefore, if we are able to prove that there exists a positive random variable $Y$ such that

$$
\mathbb{E}\left(Y^{s}\right)=\left(1+\frac{s}{\alpha}\right)^{\alpha+s} \text { for } s>-\alpha,
$$

(2) implies that $X \exp (-X / \alpha) Y$ and $X$ have the same distribution (in terms of 'arithmetic of laws', if $X$ has distribution $\gamma_{\alpha}$, the distribution of $-(X / \alpha)+\log X$ divides the distribution of $\log X$ ). Such a $Y$ does exist, as we now demonstrate.

Proposition 2

(1) There exists a probability density $f$ on $\mathbb{R}$ which is real-analytic such that

$$
s^{s}=\int_{-\infty}^{+\infty} \exp (-s x) f(x) d x \text { for } s>0 .
$$

(2) The positive random variable $Y$ such that $\log Y$ has density $\exp (-\alpha x) f(x-\log \alpha)$ satisfies

$$
\mathbb{E}\left(Y^{s}\right)=\left(1+\frac{s}{\alpha}\right)^{\alpha+s} \text { for } s>-\alpha .
$$

Proof.

(1) It is well known that $f$ is the density of a stable law with index 1 (see Berg et al. [1], p. 218): hence $f$ is real-analytic (see Lukacs [4], Theorem 5.7.5.).

(2) Consider $Z=-\log Y$. Then

$$
\begin{aligned}
\mathbb{E}\left(Y^{s}\right) & =\mathbb{E}(\exp (-s Z))=\int_{-\infty}^{+\infty} \exp (-s z) \exp (-\alpha z) f(z-\log \alpha) d z \\
& =\alpha^{-(\alpha+s)} \int_{-\infty}^{+\infty} \exp (-s x) \exp (-\alpha x) f(x) d x=\alpha^{-(\alpha+s)}(\alpha+s)^{\alpha+s}, \text { from (1). }
\end{aligned}
$$

Proof of the theorem. The 'if' part is obvious from Proposition 1(2). To prove the 'only if' part, we consider a sequence $X_{0}, Y_{1}, Y_{2}, \cdots$ of independent positive random variables such that the $Y_{j}$ have the distribution of $Y$. We introduce now the Markov chain $\left(X_{n}\right)_{n=0}^{\infty}$ on $(0,+\infty)$ defined by

$$
X_{n+1}=X_{n} \exp \left(-X_{n} / \alpha\right) Y_{n+1} \text { for } n \geqq 0 .
$$

Clearly $X \exp (-X / \alpha) Y$ and $X$ have the same distribution if and only if the distribution of $X$ is stationary for the above chain. We already know that $\gamma_{\alpha}$ is a stationary distribution; the remaining point is to see that it is the only one.

If $A$ is measurable and contained in $(0,+\infty)$, it will be called a closed set if $P(x \exp (-x / \alpha) Y \in A)=1$ for any $x$ in $A$. Since the density $f$ described in Proposition $2(1)$ is real-analytic, the density of $Y$ is strictly positive on $(0,+\infty)$ except on a countable number of points. Therefore if $A$ is a closed set, $(0,+\infty) \backslash A$ has Lebesgue measure 0 and two closed sets cannot be disjoint: this means that the chain is indecomposable and has at most one stationary distribution (see Breiman [2], Theorem 7.16).

\section{References}

[1] Berg, Ch., Christensen, J. P. R. And Ressel, P. (1984) Harmonic Analysis on Semigroups. Springer-Verlag, New York.

[2] Breiman, L. (1968) Probability. Addison-Wesley, Reading, Mass.

[3] Klamkin, M. S. (1976) On some multiple integrals. J. Math. Anal. Appl. 54, 476-479.

[4] LuKaCs, E. (1960) Characteristic Functions. Griffin, London. 